\title{
Cytokine diagnosis of pleural TB: will it stand the test of time?
}

\author{
Marc Tebruegge, 1,2,3,4 Paul T Elkington ${ }^{1}$
}

The need for better diagnostics for TB has been repeated so frequently that there is a danger of message fatigue. However, the unfortunate return of TB as the number 1 cause of death due to an infectious disease must surely serve as a further call to action for researchers, clinicians and funders to redouble our efforts. ${ }^{1}$ The fundamental issues with TB diagnosis deserve repeating: standard diagnosis of pulmonary TB relies primarily on sputum smear tests, which have limited sensitivity and specificity, and have not significantly changed for over 100 years. $^{2}$ Recent data show that staining for acid-fast bacilli remains the most commonly used diagnostic method globally. $^{3}$ The development of interferon-gamma release assays (IGRAs) and molecular amplification tests such as Xpert MTB/RIF assays has improved the landscape somewhat, but both are critically limited by cost and the need for extensive infrastructure. In addition, IGRAs do not distinguish latent from active $\mathrm{TB}$, have limited robustness and perform worse in children than in adults. $^{4-6}$ Recent data suggest that the roll-out and scale-up of the Xpert MTB/RIF assays in resource-limited settings have been far slower than anticipated. $^{7}$ Importantly, molecular assays require relatively high numbers of bacilli to achieve adequate sensitivity. The diagnosis of extrapulmonary TB is often more challenging than pulmonary TB, frequently with low bacterial loads in difficult-to-access locations. Therefore, the majority of TB diagnoses still rely on smear and culture results of specimens taken by the least invasive route, and considerable clinical acumen is often required to determine the likelihood of $\mathrm{TB}$ in the face of negative culture results.

${ }^{1}$ NIHR Biomedical Research Centre, Clinical and Experimental Sciences Academic Unit, Faculty of Medicine, University of Southampton, Southampton, UK 2Department of Paediatric Infectious Diseases and Immunology, Evelina London Children's Hospital, Guy's and St Thomas' NHS Foundation Trust, London, UK ${ }^{3}$ Great Ormond Street Institute of Child Health, University College London, London, UK

${ }^{4}$ Department of Paediatrics, University of Melbourne, Melbourne, Victoria, Australia

Correspondence to Professor Paul T Elkington, Clinical and Experimental Sciences, University of Southampton, Southampton S016 1YD, UK; p. elkington@soton.ac.uk
Data from a recent global survey suggest that microbiologists and clinicians managing patients with TB require better diagnostic tests for TB to be developed, highlighting the significant limitations of existing tests. ${ }^{3}$

On this background, the study of Wang et $a l^{8}$ published in Thorax provides a welcome addition to the literature on TB diagnosis. The group built on their previous observations that interleukin-27 (IL-27) may be a novel diagnostic marker of pleural TB, and also studied interferon- $\gamma$ $($ IFN- $\gamma$ ) and adenosine deaminase (ADA) in patients with tuberculous and non-tuberculous pleural effusions. They then studied IL-27 in a second cohort before proceeding to a meta-analysis. They report excellent test performance with a specified cut-off value, finding IL-27 more accurate than ADA and equivalent to IFN- $\gamma$. Combination of IL-27 and ADA improved specificity, but at the expense of sensitivity. The authors conclude that in high TB prevalence settings, IL-27 could be used as a rule-in test to diagnose pleural TB and in low prevalence settings as a rule-out test. These findings potentially warrant further development.

The diagnosis of pleural TB has historically been difficult, in part due to the pathophysiology of the disease. Advanced pulmonary TB is characterised by tissue destruction, hypoxia and cavitation, ${ }^{9}$ with high bacterial loads within the cavity wall, ${ }^{10}$ whereas pleural TB has a relatively low bacterial load with extensive fluid collection. ${ }^{11}$ Consequently, pleural fluid culture is positive in fewer than $50 \%$ of cases. ${ }^{12}$ Previous gold standard diagnosis has centred on pleural biopsies, as histology showing granulomas on the pleural surface has greater specificity and sensitivity than pleural fluid microscopy and culture. However, pleural biopsy with Abrams needle has become an uncommon investigation, in part due to the low incidence of TB in high-resource countries, and many clinicians with access to thoracic surgery proceed directly to video-assisted thoracoscopic surgery to obtain diagnostic tissue. ADA, an enzyme used as a marker of $T$ cell activation, showed initial promise as a novel diagnostic for pleural $\mathrm{TB},{ }^{13}$ but is not widely used in routine clinical practice in many countries.
The study by Wang et al inevitably raises further questions. First, the correct cut-off for IL-27 needs to be determined, as their meta-analysis included studies with concentrations ranging from 391 to $1007 \mathrm{ng} / \mathrm{mL}$ as diagnostic of pleural TB. Second, the utility of IL-27 will depend on the pretest probability, which itself results from the local TB epidemiology, and therefore the findings from high-incidence settings may not translate to lower incidence settings. Third, the difference between IL-27 and IFN- $\gamma$ is not so striking that it is clear which should go forward as the optimal diagnostic test. Since IFN- $\gamma$ is the read-out of IGRAs, this may drive more rapid development of next-generation IFN- $\gamma$ assays to accurately measure concentrations, which would provide an advantage in investigating this analyte further. Finally, cytokine testing is not routinely performed even in major clinical service laboratories, unlike other analytes such as $C$ reactive protein and albumin. Analysis of IFN- $\gamma$ from QuantiFERON assays is usually batched for weekly analysis in most centres, as the number of tests does not justify daily analytical runs, and so how an occasional assay for IL-27 would be performed in laboratories in high-income settings is uncertain. In low-income settings, it seems even more unlikely that such tests will become routinely available without major advances in assay technology.

A further limitation of the approach described by Wang et al is that it requires thoracentesis, which is invasive compared with assays based on blood, saliva or urine. Unfortunately, Wang et al found that IL-27 concentrations in serum did not differ between patients with $\mathrm{TB}$ and the control patients with malignant or parapneumonic effusion in their study. Also, their approach would only be useful in a small proportion of patients with $\mathrm{TB}$, as patients with isolated pleural $\mathrm{TB}$ constitute only $5 \%-10 \%$ of the total TB caseload in most settings. ${ }^{14}$ Therefore, how IL-27 as a novel diagnostic marker will move forward remains an open question. As an emerging diagnostic analyte, it faces precisely the same challenges as other putative biomarkers of $\mathrm{TB}$, such as mass spectrometry or aptamer-identified biomarkers, ${ }^{15} 16$ matrix degradation products, ${ }^{17}$ or Mycobacterium tuberculosis-specific cytokine responses in blood, ${ }^{18}$ which have shown promise in individual studies but need development and further validation.

Ultimately, the goal for the TB diagnostic field must remain to achieve the criteria previously outlined for an ideal TB test: 
available at the point of care, cheap, stable in heat and humidity, requiring minimal training, no electricity and no reagents to be added. ${ }^{19}$ This represents a very high bar, and to date has remained elusive, but such an assay will be critical in accelerating the diagnosis of TB in high-incidence settings and therefore preventing transmission and containing the ongoing pandemic. ${ }^{20}$ The study by Wang et al is a step in the right direction, adding to the literature on potential emerging diagnostic biomarkers. The challenge is now to confirm the findings in further cohorts and develop novel analytical approaches that can move diagnostics based on emerging biomarkers from the research laboratory to the clinic.

Contributors MT and PTE wrote this editorial.

Funding Medical Research Council (grant no: MR/ P023754/1).

Competing interests None declared.

Provenance and peer review Not commissioned; internally peer reviewed.

(c) Article author(s) (or their employer(s) unless otherwise stated in the text of the article) 2018. All rights reserved. No commercial use is permitted unless otherwise expressly granted.

\section{Check for updates}

To cite Tebruegge M, Elkington PT. Thorax 2018;73:206-207.

Published Online First 20 December 2017

\section{CLinked}

- http://dx.doi.org/10.1136/thoraxjnl-2016-209718

Thorax 2018;73:206-207.

doi:10.1136/thoraxjnl-2017-210918

\section{REFERENCES}

1 Wallis RS, Maeurer M, Mwaba P, et al. Tuberculosisadvances in development of new drugs, treatment regimens, host-directed therapies, and biomarkers. Lancet Infect Dis 2016;16:e34-46.

2 Dheda K, Barry CE, Maartens G. Tuberculosis. Lancet 2016:387:1211-26.

3 Amicosante M, D’Ambrosio L, Munoz M, et al. Current use and acceptability of novel diagnostic tests for active tuberculosis: a worldwide survey. J Bras Pneumol 2017:43:380-92.

4 Edwards A, Gao Y, Allan RN, et al. Corticosteroids and infliximab impair the performance of interferon- $\gamma$ release assays used for diagnosis of latent tuberculosis. Thorax 2017;72:946-9.

5 Jarvis J, Gao Y, de Graaf H, et al. Environmental temperature impacts on the performance of QuantiFERON-TB Gold In-Tube assays. J Infect 2015;71:276-80.

6 Tebruegge M, Ritz N, Curtis N, et al. Diagnostic tests for childhood tuberculosis: past imperfect, present tense and future perfect? Pediatr Infect Dis J 2015;34:1014-9.

7 Cazabon D, Suresh A, Oghor C, et al. Implementation of Xpert MTB/RIF in 22 high tuberculosis burden countries: are we making progress? Eur Respir J 2017:50:1700918

8 Wang W, Zhou Q, Zhai K, et al. Diagnostic accuracy of interleukin 27 for tuberculous pleural effusion: two prospective studies and one meta-analysis. Thorax 2018:73:240-7.
9 Belton M, Brilha S, Manavaki R, et al. Hypoxia and tissue destruction in pulmonary TB. Thorax 2016;71:1145-53.

10 Yoder MA, Lamichhane G, Bishai WR. Cavitary pulmonary tuberculosis: the holey grail of disease transmission. Current Sci 2004;86:74-81.

11 Frieden TR, Sterling TR, Munsiff SS, et al. Tuberculosis. Lancet 2003:362:887-99.

12 Ferrer J. Pleural tuberculosis. Eur Respir J 1997;10:942-7.

13 Valdés L, Alvarez D, San José E, et al. Value of adenosine deaminase in the diagnosis of tuberculous pleural effusions in young patients in a region of high prevalence of tuberculosis. Thorax 1995;50:600-3.

14 Peto HM, Pratt RH, Harrington TA, et al. Epidemiology of extrapulmonary tuberculosis in the United States, 1993-2006. Clin Infect Dis 2009:49:1350-7.

15 Achkar JM, Cortes L, Croteau P, et al. Host protein biomarkers identify active tuberculosis in HIV uninfected and co-infected individuals. EBioMedicine 2015;2:1160-8.

16 De Groote MA, Sterling DG, Hraha T, et al. Discovery and validation of a six-marker serum protein signature for the diagnosis of active pulmonary tuberculosis. J Clin Microbiol 2017;55:3057-71.

17 Walker NF, Wilkinson KA, Meintjes G, et al. Matrix degradation in human immunodeficiency virus type 1 -associated tuberculosis and tuberculosis immune reconstitution inflammatory syndrome: a prospective observational study. Clin Infect Dis 2017;65:121-32.

18 Tebruegge M, Dutta B, Donath S, et al. Mycobacteria-specific cytokine responses detect tuberculosis infection and distinguish latent from active tuberculosis. Am J Respir Crit Care Med 2015;192:485-99.

19 McNerney R, Daley P. Towards a point-of-care test for active tuberculosis: obstacles and opportunities. Nat Rev Microbiol 2011;9:204-13.

20 Elkington PT, Friedland JS. Permutations of time and place in tuberculosis. Lancet Infect Dis 2015;15:1357-60. 